\title{
Predictive factors for multidrug-resistant gram-negative bacteria among hospitalised patients with complicated urinary tract infections
}

Aina Gomila ${ }^{1,2,3^{*}}$ (D), Evelyn Shaw ${ }^{1,2,3}$, Jordi Carratalà ${ }^{1,2,3,4}$, Leonard Leibovici ${ }^{5}$, Cristian Tebé ${ }^{3}$, Irith Wiegand ${ }^{6}$, Laura Vallejo-Torres ${ }^{7}$, Joan M. Vigo ${ }^{8}$, Stephen Morris ${ }^{7}$, Margaret Stoddart ${ }^{9}$, Sally Grier ${ }^{9}$, Christiane Vank ${ }^{6}$, Nienke Cuperus ${ }^{10}$, Leonard Van den Heuvel ${ }^{10}$, Noa Eliakim-Raz ${ }^{5}$, Cuong Vuong ${ }^{6}$, Alasdair MacGowan ${ }^{9}$, Ibironke Addy ${ }^{6}$ and Miquel Pujol 1,2,3 on behalf of COMBACTE-MAGNET WP5- RESCUING Study

\begin{abstract}
Background: Patients with complicated urinary tract infections (cUTIs) frequently receive broad-spectrum antibiotics. We aimed to determine the prevalence and predictive factors of multidrug-resistant gram-negative bacteria in patients with CUTI.

Methods: This is a multicenter, retrospective cohort study in south and eastern Europe, Turkey and Israel including consecutive patients with cUTIs hospitalised between January 2013 and December 2014. Multidrug-resistance was defined as non-susceptibility to at least one agent in three or more antimicrobial categories. A mixed-effects logistic regression model was used to determine predictive factors of multidrug-resistant gram-negative bacteria cUTI.

Results: From 948 patients and 1074 microbiological isolates, Escherichia coli was the most frequent microorganism (559/1074), showing a 14.5\% multidrug-resistance rate. Klebsiella pneumoniae was second (168/1074) and exhibited the highest multidrug-resistance rate (54.2\%), followed by Pseudomonas aeruginosa (97/1074) with a 38.1\% multidrugresistance rate. Predictors of multidrug-resistant gram-negative bacteria were male gender (odds ratio [OR], 1.66; 95\% confidence interval [Cl], 1.20-2.29), acquisition of $\mathrm{CUTI}$ in a medical care facility $(\mathrm{OR}, 2.59 ; 95 \% \mathrm{Cl}, 1.80-3.71)$, presence of indwelling urinary catheter $(\mathrm{OR}, 1.44 ; 95 \% \mathrm{Cl}, 0.99-2.10)$, having had urinary tract infection within the previous year $(\mathrm{OR}, 1.89 ; 95 \% \mathrm{Cl}, 1.28-2.79)$ and antibiotic treatment within the previous 30 days $(\mathrm{OR}, 1.68 ; 95 \% \mathrm{Cl}, 1.13-2.50)$.

Conclusions: The current high rate of multidrug-resistant gram-negative bacteria infections among hospitalised patients with CUTIs in the studied area is alarming. Our predictive model could be useful to avoid inappropriate antibiotic treatment and implement antibiotic stewardship policies that enhance the use of carbapenem-sparing regimens in patients at low risk of multidrug-resistance.
\end{abstract}

Keywords: Multidrug-resistance, Complicated urinary tract infection, Gram-negative bacteria, Predictive model of multidrug-resistance gram-negative bacteria

\footnotetext{
* Correspondence: agomilagrange@gmail.com

${ }^{1}$ Department of Infectious Diseases, Hospital Universitari de Bellvitge, Institut

Català de la Salut (ICS-HUB), Feixa Llarga s/n, L'Hospitalet de Llobregat, 08907

Barcelona, Spain

${ }^{2}$ Spanish Network for Research in Infectious Diseases (REIPI RD12/0015),

Instituto de Salud Carlos III, Madrid, Spain

Full list of author information is available at the end of the article
}

(c) The Author(s). 2018 Open Access This article is distributed under the terms of the Creative Commons Attribution 4.0 International License (http://creativecommons.org/licenses/by/4.0/), which permits unrestricted use, distribution, and reproduction in any medium, provided you give appropriate credit to the original author(s) and the source, provide a link to the Creative Commons license, and indicate if changes were made. The Creative Commons Public Domain Dedication waiver (http://creativecommons.org/publicdomain/zero/1.0/) applies to the data made available in this article, unless otherwise stated. 


\section{Background}

Urinary tract infections (UTIs) are one of the most common bacterial infections [1]. Complicated urinary tract infections (cUTIs), occurring in individuals with functional or structural urinary tract abnormalities, are a leading cause of hospital admissions, hospital-acquired infections, and antibiotic use [2].

The prevalence of cUTIs is difficult to assess accurately. Data from the most recent point prevalence survey of healthcare-associated infections (HAIs) in European acute care hospitals showed that UTI was the third most common cause, accounting for $19 \%$ of estimated 3.2 million overall cases of HAIs [3]. This figure, although huge, clearly underestimates the overall cUTI incidence in Europe because it did not include patients developing cUTIs in the community and in long-term care facilities (LTCFs). In LTCFs, cUTIs occur in more than one million patients annually [4]. Aging, comorbidities, and an increasing number of invasive urologic procedures for both diagnosis and treatment have been related to this high prevalence of cUTIs in the European population.

Antibiotic resistance has become a major healthcare problem in Europe and worldwide [5, 6]. Currently, multidrug-resistant (MDR) gram-negative bacteria (GNB) pose a threat in hospitals and nursing homes [7]. According to the recent Annual Report of the European Antimicrobial Resistance Surveillance Network (EARS-Net) [8], MDR rates showed large variations across Europe, being higher in southern and south-eastern Europe than in northern Europe. Patients with suspected cUTIs are frequently treated empirically with broad-spectrum antibiotics. Developing a model that helps select patients at high risk for MDR could be useful when choosing empirical antibiotic regimens and in antibiotic stewardship policies.

Considering the lack of contemporary data on hospitalised patients with cUTIs, we aimed to determine the prevalence of MDR among hospitalised patients with cUTIs in countries with high MDR-GNB prevalence and develop a predictive model to determine the risk of MDR-GNB infections, which would be useful to select more targeted antibiotic regimens avoiding the frequent treatment with broad-spectrum antibiotics.

\section{Methods}

\section{Study design}

The COMBACTE-MAGNET, WP5 RESCUING Study was a multicenter, retrospective, observational cohort study including hospitalised patients with cUTI from January 2013 to December 2014. Data was collected from patients who were diagnosed with cUTI as the primary cause of hospitalisation and from patients who were hospitalised for other reasons but who developed cUTIs during their hospitalization [9]. This study conformed to the STROBE guidelines for reporting observational studies [10].

\section{Setting and patients}

The study was conducted in Bulgaria (2 hospitals), Greece (2 hospitals), Hungary (3 hospitals), Israel (3 hospitals), Italy (3 hospitals), Romania (2 hospitals), Spain (3 hospitals) and Turkey ( 2 hospitals). Patients were identified by searching for the appropriate International Classification of Diseases (ICD)-9 Clinical Modification (CM) or ICD-10 CM Codes $[11,12]$ at discharge from hospital (diagnoses are detailed in Additional file 1). All patients who met the criteria for cUTI were selected for data collection. In order to avoid selection bias, each hospital included 50 to 60 consecutive patients with cUTI until achieving the total estimated sample size of 1000 patients.

Complicated urinary tract infection inclusion criteria followed the Food and Drug Administration (FDA) guidance on cUTI [13], and consisted on:

- Patients with UTI and at least one of the following underlying conditions: a) indwelling urinary catheter; b) urinary retention (at least $100 \mathrm{~mL}$ of residual urine after voiding); c) neurogenic bladder; d) obstructive uropathy (e.g., nephrolithiasis, fibrosis); e) renal impairment caused by intrinsic renal disease (estimated glomerular filtration rate $<60 \mathrm{~mL} / \mathrm{min}$ ); f) renal transplantation; g) urinary tract modifications, such as an ileal loop or pouch; or h) pyelonephritis.

- And at least one of the following signs or symptoms: a) chills or rigors associated with fever or hypothermia (temperature $>38{ }^{\circ} \mathrm{C}$ or $<36{ }^{\circ} \mathrm{C}$ ); b) flank pain (pyelonephritis) or pelvic pain (cUTI); c) dysuria, urinary frequency, or urinary urgency; or d) costovertebral angle tenderness on physical examination.

- And urine culture with $\geq 10^{5}$ colony-forming units/ $\mathrm{mL}$ of uropathogen (no more than two species) or;

- At least one blood culture growing possible uropathogens (no more than two species) with no other evident site of infection.

The exclusion criteria were as follows: a) patients aged < 18 years, b) diagnosis of prostatitis according to FDA guidance, c) polymicrobial infections including Candida spp., d) polymicrobial infections including more than two bacterial species, or e) cUTI with Candida spp. as sole uropathogen, d) patients with uncomplicated cystitis.

If a patient had more than one episode of cUTI during the same hospitalisation, only the first episode was included.

\section{Data collection and validation}

Data on demographic characteristics, comorbidities, place of acquisition of infection, signs and symptoms of infection, laboratory and microbiology, imaging tests, management of infection including antibiotic therapy and interventional procedures, details of discharge and outcome of infection, including death if applicable, were reviewed by 
professionals who received web-database training sessions. For data collection, an access-controlled web-based electronic case report form was used. At each site, a screening log was kept of the patients with infections detected according to the ICD codes, detailing the excluded patients and the reasons for exclusion. To confirm data quality, study sites were monitored and audited by a contract research organization (CRO) from Utrecht, Netherlands.

\section{Definitions}

Acquisition of cUTI in a medical care facility was considered if it was:

- Hospital-acquired: if it started $\geq 48 \mathrm{~h}$ after hospital admission.

- Healthcare-associated: if it was detected at hospital admission or within the first $48 \mathrm{~h}$ of hospitalization, with the patient fulfilling any of the following criteria: 1) receiving intravenous therapy, wound care, or specialized nursing care at home in the previous 30 days; 2) admission in the hospital or haemodialysis ward or receiving intravenous chemotherapy in the previous 30 days; 3 ) hospitalization for $\geq 2$ days in the previous 90 days; 4) residence in a long-term care facility; 5) underwent invasive urinary procedure within the previous 30 days; or 6) having a long-term indwelling urinary catheter.

We used the following categories for cUTIs:

- UTI related to indwelling urinary catheterization, including long-term, short-term, or intermittent catheterization

- Pyelonephritis with no other urinary tract modification, defined as sepsis, flank pain or costovertebral angle tenderness

- UTI related to anatomical urinary tract modification, including any urinary diversion procedure, nephrostomy or stents, or renal transplants

- UTI related to obstructive uropathy, including any obstruction intrinsic or extrinsic to the urinary tract, such as lithiasis, tumor, ureteral herniation, or prostate hyperplasia

- UTI related to other events that do not fall under any other category

Multidrug resistance was defined according to an international expert proposal by Magiorakos et al. [14], as non-susceptibility to at least one agent in three or more antimicrobial categories (extended-spectrum penicillins, carbapenems, cephalosporins, aminoglycosides, and fluoroquinolones). Extensively drug-resistance (XDR) was defined as non-susceptibility to at least one agent in all but two or fewer antimicrobial categories (i.e., bacterial isolates remaining susceptible to only one or two categories) tested for a determined microorganism.

\section{Outcomes}

The primary outcome was the presence of MDR, as previously defined.

Secondary outcomes included the following:

- Estimation of the MDR prevalence in each country and participating hospital

- Definition of the most prevalent microbiology according to source of infection

- Assessment of the resistance rate of the main GNB to the different antimicrobial classes

\section{Statistical methods}

The chi-square or Fisher's exact test was used to compare categorical data, and Student's t-test or the Mann-Whitney $\mathrm{U}$ test to compare continuous data, as appropriate. The quantile-quantile normality plot and Kolmogorov-Smirnov test were used to assess whether a continuous variable was normally distributed.

\section{Predictive model of MDR in patients with cUTI}

Countries and hospitals presented a non-homogeneous MDR baseline risk. To account for such variations, a mixed-effects logistic regression model to predict the risk of MDR in patients with cUTIs, including all different epidemiological and clinical variables, was built using hospitals as clusters. First, a stepwise selection method based on the Akaike Information Criterion was performed to identify variables that explained the bulk of MDR infections. Adequacy of the final model was assessed by collinearity, influential observations, and residuals. To evaluate discrimination properties, the Hosmer-Lemeshow goodness-of-fit test was used. Moreover, the bootstrapping resampling method was used to improve the robustness of estimated standard errors. Results were given as odds ratios (OR) and 95\% confidence intervals $(95 \% \mathrm{CI})$. All tests were two-tailed, and a $p$-value of $<0.05$ was considered statistically significant.

All data were analyzed using $R$ software (2017). R Foundation for Statistical Computing, Vienna, Austria.

\section{Results}

Patients' epidemiological characteristics and univariate analysis of MDR-GNB

Fifty-two cases were excluded due to lack of information on the presence of MDR, leaving a final sample of 948 patients. Among them, 1074 bacterial isolates were obtained.

The patients' clinical characteristics are shown in Table 1. Females comprised $56 \%$, the mean age was $65.8 \pm 18.2$ years, $34.4 \%$ were admitted due to conditions other than cUTIs, $17.4 \%$ came from LTCFs, and $46 \%$ were functionally dependent. Factors associated with MDR by univariate 
Table 1 Patients' epidemiological characteristics and univariate analysis of multidrug-resistance in gram-negative bacteria

\begin{tabular}{|c|c|c|c|c|}
\hline & $\begin{array}{l}\text { Entire Cohort } \\
(n=948)\end{array}$ & $\begin{array}{l}\text { Susceptible } \\
(n=691)\end{array}$ & $\begin{array}{l}\text { MDR } \\
(n=257)\end{array}$ & $p$-Value \\
\hline Male gender, n (\%) & $420(44.3)$ & $270(39.1)$ & $150(58.4)$ & $<0.001$ \\
\hline Mean age $(S D)$, years & $65.8(18.2)$ & $65.6(18.6)$ & $66.5(16.8)$ & 0.526 \\
\hline Elective admission, n (\%) & $141(14.9)$ & $97(14)$ & $44(17.1)$ & 0.236 \\
\hline Admission reason: conditions other than cUTI, n (\%) & $326(34.4)$ & $214(31)$ & $112(43.6)$ & $<0.001$ \\
\hline Place of residency: long-term care facility, n (\%) & $165(17.4)$ & $98(14.2)$ & $67(26.1)$ & $<0.001$ \\
\hline \multicolumn{5}{|l|}{ Underlying disease, n (\%) } \\
\hline Acute myocardial infarction & $79(8.3)$ & $56(8.1)$ & $23(8.9)$ & 0.676 \\
\hline Congestive heart failure & $182(19.2)$ & $134(19.4)$ & $48(18.7 \%)$ & 0.804 \\
\hline Peripheral vascular disease & $70(7.4)$ & $55(8)$ & $15(5.8)$ & 0.267 \\
\hline Cerebrovascular disease & $182(19.2)$ & $122(17.7)$ & $60(23.3)$ & 0.048 \\
\hline Dementia & $130(13.7)$ & $93(13.5)$ & $37(14.4)$ & 0.709 \\
\hline Chronic pulmonary disease & $135(14.2)$ & $91(13.2)$ & $44(17.1)$ & 0.122 \\
\hline Connective tissue disease & $21(2.2)$ & $15(2.2)$ & $6(2.3)$ & 0.879 \\
\hline Peptic ulcer & $46(4.9)$ & $34(4.9)$ & $12(4.7)$ & 0.873 \\
\hline Diabetes mellitus & $250(26.4)$ & $186(26.9)$ & $64(24.9)$ & 0.531 \\
\hline Chronic kidney disease & $263(27.7)$ & $191(27.6)$ & $72(28)$ & 0.909 \\
\hline Hemiplegia & $86(9.1)$ & $58(8.4)$ & $28(10.9)$ & 0.233 \\
\hline Leukaemia & $9(0.9)$ & $6(0.9)$ & $3(1.2)$ & 0.673 \\
\hline Lymphoma & $13(1.4)$ & $11(1.6)$ & $2(0.8)$ & 0.338 \\
\hline Chronic liver disease & $50(5.3)$ & $35(5.1)$ & $15(5.8)$ & 0.637 \\
\hline Solid tumour & $114(12.3)$ & $75(11.1)$ & $39(15.4)$ & 0.075 \\
\hline Metastatic tumour & $47(5)$ & $35(5.1)$ & $12(4.7)$ & 0.803 \\
\hline Valvulopathy & $88(9.3)$ & $69(10)$ & $19(7.4)$ & 0.221 \\
\hline HIV infection & $10(1.1)$ & $8(1.2)$ & $2(0.8)$ & 0.611 \\
\hline Charlson index $\geq 3, \mathrm{n}(\%)$ & $418(44.1)$ & $299(43.3)$ & $119(46.3)$ & 0.403 \\
\hline Organ transplant, n (\%) & $65(6.9)$ & $45(6.5)$ & $20(7.8)$ & 0.492 \\
\hline Immunosuppression, n (\%) & $94(9.9)$ & $64(9.3)$ & $30(11.7)$ & 0.270 \\
\hline Steroids, n (\%) & $68(7.2)$ & $46(6.7)$ & $22(8.6)$ & 0.313 \\
\hline Functional capacity: dependent, n (\%) & $436(46.1)$ & $298(43.3 \%)$ & $138(53.9)$ & 0.003 \\
\hline Prior UTI (within the previous year), n (\%) & $247(26.1)$ & $167(24.2)$ & $80(31.2)$ & 0.027 \\
\hline Prior antibiotics (within the previous 30 days), n (\%) & $190(20.1)$ & $120(17.4)$ & $70(27.6)$ & 0.001 \\
\hline Prior quinolone & $64(6.8)$ & $38(5.5)$ & $26(10.2)$ & 0.010 \\
\hline Prior Penicillin & $55(5.8)$ & $35(5.1)$ & $20(7.9)$ & 0.103 \\
\hline Prior cephalosporin & $42(4.4)$ & $27(3.9)$ & $15(5.9)$ & 0.188 \\
\hline Prior Carbapenem & $22(2.3)$ & $10(1.4)$ & $12(4.7)$ & 0.003 \\
\hline Prior other antibiotics & $51(5.4)$ & $31(4.5)$ & $20(7.9)$ & 0.042 \\
\hline Acquisition in a medical care facility, $\mathrm{n}(\%)$ & $410(43.2)$ & $244(35.3)$ & $166(64.6)$ & $<0.001$ \\
\hline \multicolumn{5}{|l|}{ Source of cUTI, n (\%) } \\
\hline Indwelling urinary catheterisation & $308(32.5)$ & $189(27.4)$ & $119(46.3)$ & $<0.001$ \\
\hline Pyelonephritis with normal tract anatomy & $196(20.7)$ & $164(23.7)$ & $32(12.5)$ & $<0.001$ \\
\hline Obstructive uropathy & $152(16)$ & $114(16.5)$ & $38(14.8)$ & 0.523 \\
\hline Urinary tract diversion & $84(8.9)$ & $64(9.3)$ & $20(7.8)$ & 0.476 \\
\hline
\end{tabular}


Table 1 Patients' epidemiological characteristics and univariate analysis of multidrug-resistance in gram-negative bacteria (Continued)

\begin{tabular}{cllll}
\hline & $\begin{array}{l}\text { Entire Cohort } \\
(n=948)\end{array}$ & $\begin{array}{l}\text { Susceptible } \\
(n=691)\end{array}$ & $\begin{array}{l}\text { MDR } \\
(n=257)\end{array}$ & $p$-Value \\
\hline Other & $208(21.9)$ & $160(23.2)$ & $48(18.7)$ & 0.139 \\
Shock/severe sepsis, $\mathrm{n}(\%)$ & $140(15.9)$ & $104(16.2)$ & $36(14.9)$ & 0.635 \\
\hline
\end{tabular}

MDR multidrug resistance, $S D$ standard deviation, $C U T I$ complicated urinary tract infection, HIV human immunodeficiency virus, UTI urinary tract infection

analysis were male gender, admission due to reasons other than cUTIs, residing in LTCF, dependent functional capacity, UTI within the previous year, antibiotic treatment within the previous 30 days, acquisition of CUTI in a medical care facility, and presence of an indwelling urinary catheter.

\section{Most frequent bacterial aetiology and patterns of antimicrobial resistance}

Of all bacterial isolates $(n=1074)$, the most frequent was Escherichia coli, isolated in $52 \%$ of samples, followed by Klebsiella pneumoniae in $15.6 \%$, Pseudomonas aeruginosa in 9\%, Proteus mirabilis in 7.3\%, and Enterococcus spp. in $3.2 \%$. Only these 5 bacteria were evaluated due to their clinical significance. Escherichia coli was mainly related to pyelonephritis with normal urinary tract (76.5\%), while $K$. pneumoniae was more frequently associated with urinary tract diversion (22.6\%). Pseudomonas aeruginosa, P. mirabilis and Enterococcus spp. were significantly related to the presence of an indwelling urinary catheter (18.8\%, 25.6\% and 5.8\% respectively) (Table 2).

Significant differences in MDR rate occurred between the different participating hospitals, ranging from $<20 \%$ in some countries such as Hungary and Spain to almost $60 \%$ in other countries such as Bulgaria and Greece (Fig. 1a). The MDR rates by hospital varied in accordance with the country's trend (Fig. 1b).

The antimicrobial resistance patterns according to the most frequent GNB are shown in Table 3. Escherichia coli had a fluoroquinolone resistance rate of $39.5 \%$, a third-generation cephalosporin (3GC) resistance rate of $24.2 \%$ and a MDR rate of $14.5 \%$. Klebsiella pneumoniae exhibited the highest MDR rate $(54.2 \%)$, followed by $P$. aeruginosa (38.5\%) and P. mirabilis (24.1\%). By antibiotic class, fluoroquinolones had the highest resistance rates $(39.5 \%$ in E. coli, $56.5 \%$ in K. pneumoniae, $42.1 \%$ in $P$. aeruginosa, and $55.7 \%$ in $P$. mirabilis), followed by 3GC and aminoglycosides (Table 3). Resistance to carbapenems was $32.6 \%$ in $P$. aeruginosa, $19.6 \%$ in $K$. pneumoniae and $2.3 \%$ in E. coli.

\section{Predictive model of MDR-GNB in patients with cUTIs}

Identified predictive factors for MDR risk are reported in Table 4. The resulting equation and an illustrative example for calculating MDR-GNB risk are described in Additional file 1 . The proposed model had good discrimination for MDR prediction, with a 0.80 statistic (area under the receiver operating characteristic curve) (Fig. 2). Calibration was also excellent, with a good observed/expected ratio of MDR risk by deciles of predicted risk (Fig. 3a) and by hospital (Fig. 3b).

The factors that best predicted the bulk of MDR presence were male gender (odds ratio [OR], 1.66; 95\% confidence interval [CI], 1.20-2.29), acquisition of cUTI in a medical care facility (OR, 2.59; 95\% CI, 1.80-3.71), presence of an indwelling urinary catheter (OR, 1.44; 95\% CI, 0.99-2.10), having a UTI within the previous year (OR, 1.89; 95\% CI, 1.28-2.79), and antibiotic treatment within the previous 30 days (OR, 1.68; 95\% CI, 1.13-2.50) (Table 4).

Table 2 Most frequent bacterial aetiology of complicated urinary tract infections according to source of infection (sources $=948$, isolations $=1074)$

\begin{tabular}{|c|c|c|c|c|c|}
\hline Source $(n=948)$ & $\begin{array}{l}\text { E. coli } \\
n=559(52 \%)\end{array}$ & $\begin{array}{l}\text { K. pneumoniae } \\
n=168(15.6 \%)\end{array}$ & $\begin{array}{l}P . \text { aeruginosa } \\
n=97(9 \%)\end{array}$ & $\begin{array}{l}\text { P. mirabilis } \\
n=79(7.3 \%)\end{array}$ & $\begin{array}{l}\text { Enterococcus spp. } \\
n=34(3.2 \%)\end{array}$ \\
\hline $\begin{array}{l}\text { Indwelling urinary catheterisation } \\
(n=308), \mathrm{n}(\%)\end{array}$ & $124(40.3 \%)$ & $63(20.4 \%)$ & $58(18.8 \%)$ & $40(25.6 \%)$ & $18(5.8 \%)$ \\
\hline $\begin{array}{l}\text { Pyelonephritis with normal tract } \\
\text { anatomy }(n=196), \mathrm{n}(\%)\end{array}$ & $150(76.5 \%)$ & $25(12.7 \%)$ & $4(2.0 \%)$ & $13(6.6 \%)$ & $0(0.0)$ \\
\hline Obstructive uropathy $(n=152), \mathrm{n}(\%)$ & $98(64.4 \%)$ & $26(17.1 \%)$ & $12(7.9 \%)$ & $11(7.2 \%)$ & $5(3.3 \%)$ \\
\hline Urinary tract diversion $(n=84), \mathrm{n}(\%)$ & $48(57.1 \%)$ & $19(22.6 \%)$ & $10(11.9 \%)$ & $2(2.4 \%)$ & $4(4.8 \%)$ \\
\hline Others $(n=208), \mathrm{n}(\%)$ & $139(66.8 \%)$ & $35(16.8 \%)$ & $13(6.2 \%)$ & $13(6.2 \%)$ & $7(3.4 \%)$ \\
\hline
\end{tabular}

E. coli, Escherichia coli; K. pneumoniae, Klebsiella pneumoniae; P. aeruginosa, Pseudomonas aeruginosa; P. mirabilis, Proteus mirabilis; Enterococcus spp., Enterococcus species. First column include all sources of infection $(n=948)$, and first raw include the five more frequent bacteria taking as denominator the total number of isolations $(n=1074)$. All other isolates up to the total number are not included in the table. Denominators in central boxes are the total number of each row (sources) 




Fig. 1 Cumulative multidrug-resistant gram-negative bacteria incidence with 95\% confidence interval by country (a) and by hospital (b). Figure 1 shows the cumulative incidence with 95\% confidence interval of multidrug-resistant (MDR) gram-negative bacteria in complicated urinary tract infection observed in each participating country (a) and in each participating hospital (b). Hosp. Univ. 12 de Octubre: Hospital Universitario 12 de Octubre, Hosp. Univ. Virgen Macarena: Hospital Universitario Virgen de la Macarena, Hosp. Univ. Bellvitge: Hospital Universitari de Bellvitge

\section{Discussion}

This large, multicenter, retrospective cohort study of hospitalised patients with cUTIs provides a comprehensive update about antibiotic resistance in countries with high MDR incidence. In this cohort, $K$. pneumoniae had the highest MDR rate among all the GNB analysed, and fluoroquinolones had the highest resistance rates. We developed a model to predict the risk of cUTIs caused by
MDR organisms, in order to avoid inappropriate treatment and help establish antibiotic stewardship policies.

In our cohort, $E$. coli continues to be the most frequent cause of cUTI, as previously observed [15, 16]. Although it was associated with low MDR levels, it showed a fluoroquinolone resistance rate of almost $40 \%$ and a $3 G C$ resistance rate of $24 \%$. Previous studies already described an increased resistance rate of $E$. coli to fluoroquinolones and 
Table 3 Patterns of antimicrobial resistance to main antibiotic groups by the four most frequent gram-negative bacteria

\begin{tabular}{|c|c|c|c|c|c|c|c|}
\hline & $\begin{array}{l}\text { AMG-R } \\
\mathrm{n}(\%)\end{array}$ & $\begin{array}{l}\text { FQ-R } \\
\text { n (\%) }\end{array}$ & $\begin{array}{l}\text { 3GC-R } \\
\mathrm{n}(\%)\end{array}$ & $\begin{array}{l}\mathrm{P} / \mathrm{T}-\mathrm{R} \\
\mathrm{n}(\%)\end{array}$ & $\begin{array}{l}\text { CARB-R } \\
\mathrm{n}(\%)\end{array}$ & $\begin{array}{l}\text { MDR } \\
\mathrm{n}(\%)\end{array}$ & $\begin{array}{l}\text { XDR } \\
\mathrm{n}(\%)\end{array}$ \\
\hline $\begin{array}{l}\text { E. coli } \\
(n=559)\end{array}$ & $108(19.3)$ & $221(39.5)$ & $135(24.2)$ & $57(10.2)$ & $13(2.3)$ & 81 (14.5) & $2(0.4)$ \\
\hline $\begin{array}{l}\text { K. pneumoniae } \\
(n=168)\end{array}$ & 77 (45.8) & 95 (56.5) & 98 (58.3) & 64 (38.1) & 33 (19.6) & 91 (54.2) & $23(13.7)$ \\
\hline $\begin{array}{l}\text { P. aeruginosa } \\
(n=97)\end{array}$ & 36 (37.9) & $40(42.1)$ & $47(49.5)$ & 30 (31.6) & 31 (32.6) & $36(38.5)$ & $16(16.8)$ \\
\hline $\begin{array}{l}\text { P. mirabilis } \\
(n=79)\end{array}$ & $29(36.7)$ & 44 (55.7) & $20(25.4)$ & $9(11.4)$ & $4(5.0)$ & $19(24.1)$ & $1(1.3)$ \\
\hline
\end{tabular}

$A M G-R$ aminoglycoside-resistant, $F Q-R$ fluoroquinolone-resistant, $3 G C-R$ third-generation cephalosporin-resistant, $P / T-R$ piperacillin/tazobactam-resistant, $C A R B-R$ carbapenem-resistant, MDR multidrug-resistant, XDR extensively drug-resistant, E. coli, Escherichia coli; K. pneumoniae, Klebsiella pneumoniae; $P$. aeruginosa,

Pseudomonas aeruginosa; P. mirabilis, Proteus mirabilis

trimethoprim-sulfamethoxazole, precluding their use as empiric treatment in mild and severe infections [16, 17]. Similar to our results, the Study for Monitoring Antimicrobial Resistance Trends (SMART) in the United States showed a $35 \%$ resistance rate of $E$. coli to ciprofloxacin [18]. This high fluoroquinolone resistance rate contrasts with the $20 \%$ reported by the EARS-Net in 2016 [8]. However, the rate was obtained by including northern European countries that had low antimicrobial resistance rates. On the contrary, the south-eastern countries showed rates similar to those observed in our study. Besides, the EARS-Net included only invasive isolates, a sample profile quite different to ours. MDR rates similar to our results were also observed in the Asia-Pacific region [19].

In our cohort, $K$. pneumoniae was the second most frequent microorganism, showing a remarkably carbapenemresistance rate of almost $20 \%$ and having ileal loop or urinary diversion as the most frequent source of infection.

Table 4 Predictive model of multidrug-resistant gram-negative bacteria in patients with complicated urinary tract infection: a mixed-effects logistic regression model

\begin{tabular}{llll}
\hline Factors & OR & $95 \% \mathrm{Cl}$ & $p$-Value \\
\hline (Intercept) & 0.1 & $0.06-0.16$ & $<0.001$ \\
Gender (male) & 1.66 & $1.20-2.29$ & 0.002 \\
Acquisition in a medical facility & 2.59 & $1.80-3.71$ & $<0.001$ \\
Indwelling urinary catheter & 1.44 & $0.99-2.10$ & 0.06 \\
UTI within the previous year & 1.89 & $1.28-2.79$ & 0.001 \\
Antibiotics within the previous 30 days & 1.68 & $1.13-2.50$ & 0.011 \\
\hline
\end{tabular}

$O R$ odds ratio, $C l$ confidence interval, UTI urinary tract infection

Potential predictors included in the predictive model were age, sex, source of infection, place of residency, functional capacity score, personal history of myocardial infarction, congestive heart failure, peripheral vascular disease, cerebrovascular disease, dementia, chronic pulmonary disease, ulcer disease, diabetes mellitus, chronic kidney disease, hemiplaegia, solid tumor, liver disease, metastatic tumor, Charlson score, infection acquisition site, presence of indwelling urinary catheter, urinary retention, organ transplant, kidney organ transplant, immunosuppressive therapy, active chemotherapy, corticosteroid therapy, UTI within the previous year, previous 30-day antibiotic treatment (including previous treatment with quinolones, penicillins, cephalosporins, carbapenems, and other antibiotics), infection severity, neurogenic bladder, obstructive uropathy, other urinary tract modification, and chronic renal impairment
The countries with the highest rate of carbapenem-resistant K. pneumoniae were Greece and Turkey, while those with the lowest were Spain and Hungary. This study did not analyse the type of resistance mechanisms present in Enterobacteriaceae; nevertheless, phenotypic resistance to carbapenems commonly results from acquiring carbapenemases that affect even the latest generations of penicillins and cephalosporins, in addition to other antibiotic families such as aminoglycosides and fluoroquinolones. The European survey of carbapenemase-producing Enterobacteriaceae (EuSCAPE), performed in 2013-2014 in Europe, Turkey, and Israel, showed that K. pneumoniae and

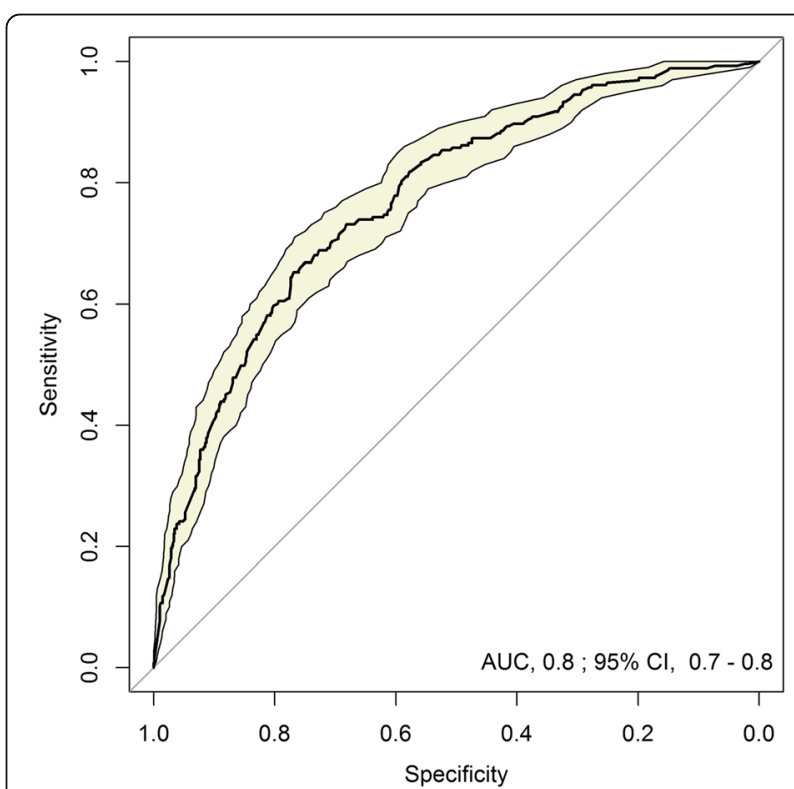

Fig. 2 Receiver operating characteristic curve of the predictive model of multidrug-resistance in gram-negative bacteria. Figure 2 shows the evaluation of the discriminative power of the mixedeffects logistic regression predictive model for multidrug-resistant gram-negative bacteria among patients with complicated urinary tract infection by the receiver operating characteristic curve using observed multidrug-resistance incidence as the gold standard. AUC, area under the curve; $\mathrm{Cl}$, confidence interval 




E. coli produced carbapenemases, mainly KPC-type and OXA-48-like, in the countries represented in our study [20]. However, $29 \%$ of $K$. pneumoniae isolates had unidentified mechanisms of carbapenem resistance, and almost $10 \%$ of $K$. pneumoniae isolates were resistant to all antibiotics tested, consistent with our findings.

$P$. aeruginosa isolates showed a carbapenem-resistance rate that reached $32 \%$. In this case, the presence of a 
urinary catheter was the most frequently associated factor. Countries with the greatest rates of carbapenem-resistant $P$. aeruginosa included Italy and Turkey, while those with the lowest rates were Israel and Hungary. The mechanisms of MDR in P. aeruginosa have been related to the production of cephalosporinases, combined with mutations that decrease carbapenem permeability of the bacterial cell wall [21-23]. The selective antibiotic pressure caused by broad-spectrum antibiotics favours the emergence of MDR strains, and once it is produced, its reversion is very slow [24].

We have developed and internally validated a clinical predictive model for hospitalised patients with suspected cUTIs that helps determine the risk of MDR-GNB infections, considering the country's baseline risk. This model may be useful in reducing inappropriate empirical antibiotic treatment that leads to poor clinical outcomes in these patients [25]. It may also help implement antibiotic stewardship programs that enhance the use of carbapenem-sparing antibiotic regimens in patients at low risk for MDR $[24,26]$. The severity of infection based on physician's clinical judgement and severity scores needs to be assessed, since non-severe cUTI will probably benefit more from receiving treatment based on susceptibility testing [27]. Importantly, however, more severe cases with potentially serious consequences of treatment failure could benefit from applying our model.

The most reliable factor that predicted MDR was the acquisition of cUTIs in medical care facilities, mostly LTCFs. Most patients admitted to LTCFs are old, have comorbidities, and are functionally dependent. These patients frequently receive repeated courses of antibiotics for various reasons, including cUTIs. Thus, LTCFs have been identified as important reservoirs of MDR-GNB [28]. Besides patients having had a UTI within the previous year and having received antibiotics within the previous month, other predictive factors for MDR identified by our model have been also described by other authors $[29,30]$. All of them reflect high cumulative exposure to antibiotics and, consequently, selection of MDR endogenous flora.

Male UTI is usually considered complicated due to the more complex urinary tract anatomy. This implies longer antibiotic treatments and frequent relapse of infection, resulting in repeated antibiotic exposure and higher risk of MDR [31].

The presence of a urinary catheter has been associated with a higher risk of UTI $[32,33]$ and infections caused by microorganisms with higher intrinsic resistance, such as $P$. aeruginosa and Enterococcus spp. [1]. Our study reaffirmed this observation since $P$. aeruginosa was significantly associated with urinary catheter use. The catheter inhibits the defence mechanisms of the urinary tract epithelium against bacteria and facilitates the rapid invasion of the bladder by microorganisms colonizing the device. The urinary catheter also promotes the development of bacterial biofilm, where antibiotics do not achieve significant concentrations [34].

The main limitation of this study is that the model has been validated in a group of hospitals from south and eastern Europe, Turkey and Israel and the results may not be generalizable to other populations. Therefore, further external validation is necessary to confirm our results. Also, the retrospective design and approach for identifying cases could have led to underestimate non-severe cases occurring in patients admitted due to other reason than cUTI and who developed cUTI during the hospitalisation. On the other hand, difficult to treat MDR-GNB cUTIs could have been more easily identified. The main strength of the study is its large-scale, multicenter, and multinational design including 948 patients and the case-validation system. Furthermore, the effect of possible differences in MDR baseline risk by each hospital on the main outcome was considered to create the predictive model.

\section{Conclusions}

The current high rate of MDR-GNB infections among hospitalised patients with cUTIs is alarming in south and eastern Europe, Turkey and Israel. A high MDR rate has been observed among $K$. pneumoniae and $P$. aeruginosa isolates. Our study developed a predictive model that could be useful in determining the risk for MDR-GNB cUTI, with the purpose of targeting patients at high risk with broad-spectrum antibiotics and guiding the implementation of antibiotic stewardship policies that enhance the use of carbapenem-sparing antibiotic regimens in patients at low risk for MDR-GNB.

\section{Additional file}

Additional file 1: Supplementary material. (DOCX 24 kb)

\section{Abbreviations}

95\%Cl: 95\% confidence interval; CRO: Contract research organization; CUTIs: Complicated urinary tract infections; EARS-Net: European Antimicrobial Resistance Surveillance Network; EuSCAPE: European survey of

carbapenemase-producing Enterobacteriaceae; FDA: Food and Drug Administration; GNB: Gram-negative bacteria; HAls: Healthcare-associated infections; ICD-9 (-10) CM: International Classification of Diseases-9 (-10) Clinical Modification; KPC: Klebsiella pneumoniae carbapenemase;

LTCFs: Long-term care facilities; MDR: Multidrug-resistance; OR: Odds ratio; OXA: Oxacillinase; SMART: Study for Monitoring Antimicrobial Resistance Trends; STROBE: Strengthening the Reporting of Observational studies in Epidemiology; UTIs: Urinary tract infections; XDR: Extensively drug-resistance

\section{Acknowledgements}

COMBACTE-MAGNET, RESCUING Study Group members: Tanya Babitch, Dora Tancheva, Rossitza Vatcheva-Dobrevska, Sotirios Tsiodras, Emmanuel Roilides, Istvan Várkonyi, Judit Bodnár, Aniko Farkas, Yael Zak-Doron, Yehuda Carmeli, Emanuele Durante Mangoni, Cristina Mussini, Nicola Petrosillo, Andrei Vata, Adriana Hristea, Julia Origüen, Jesus Rodriguez-Baño, Arzu Yetkin, and Nese Saltoglu. 


\section{Funding}

This research project received support from the Innovative Medicines Initiative Joint Undertaking under grant agreement $n^{\circ} 115523$ | 115620 | 115737 resources of which are composed of financial contribution from the European Union Seventh Framework Programme (FP7/2007-2013) and EFPIA companies in kind contribution. The research leading to these results was conducted as part of the COMBACTE-MAGNET consortium. For further information please refer to WWW.COMBACTE.COM.

\section{Availability of data and materials}

The datasets used and/or analysed during the current study are available from the corresponding author on reasonable request.

\section{Authors' contributions}

$A G, L L$ and MP conceived and designed the study. AG, JC and MP were major contributors in writing the manuscript. ES, LL, IW, CV, CV, IA participated in the design of the study and coordination and helped to draft the manuscript. CT performed the statistical analysis of data. All authors read and approved the final manuscript.

\section{Ethics approval and consent to participate}

This study was approved by the local research ethics committee of each site. The processing of patients' personal data was anonymised and complied with local data protection legislations and with the European Union Data Protection Directive (95/46/EC). As no individual data is published as well as no intervention was performed to patients, patient consent was waived by the Ethics Committee.

\section{Consent for publication}

No applicable.

\section{Competing interests}

Authors IA, CV, IW, and CV belong to EFPIA (European Federation of Pharmaceutical Industries and Association) member companies in the IMI JU and costs related to their part in the research were carried by the respective company as in kind contribution under the IMI JU scheme. Other authors declare no potential conflicts.

\section{Publisher's Note}

Springer Nature remains neutral with regard to jurisdictional claims in published maps and institutional affiliations.

\section{Author details \\ ${ }^{1}$ Department of Infectious Diseases, Hospital Universitari de Bellvitge, Institut Català de la Salut (ICS-HUB), Feixa Llarga s/n, L'Hospitalet de Llobregat, 08907 Barcelona, Spain. ${ }^{2}$ Spanish Network for Research in Infectious Diseases (REIPI RD12/0015), Instituto de Salud Carlos III, Madrid, Spain. ${ }^{3}$ Institut d'Investigació Biomèdica de Bellvitge (IDIBELL), Feixa Llarga s/n, L'Hospitalet de Llobregat, 08907 Barcelona, Spain. ${ }^{4}$ University of Barcelona, Barcelona, Spain. ${ }^{5}$ Department of Medicine E, Beilinson Hospital, Rabin Medical Center, Petah Tikva; Sackler Faculty of Medicine, Tel Aviv University, Tel Aviv, Israel. ${ }^{6}$ AiCuris Anti-infective Cures GmbH, Wuppertal, Germany. ${ }^{7} \mathrm{UCL}$ Department of Applied Health Research, University College London, London, UK. ${ }^{8}$ Informatics Unit, Fundació Institut Català de Farmacologia, Barcelona, Spain. ${ }^{9}$ Department of Medical Microbiology, Southmead Hospital, North Bristol NHS Trust, Bristol, UK. ${ }^{10}$ Julius Center for Health Sciences and Primary Care, University Medical Center Utrecht, Utrecht, Netherlands.}

\section{Received: 24 July 2018 Accepted: 29 August 2018}

Published online: 14 September 2018

\section{References}

1. Flores-Mireles AL, Walker JN, Caparon M, Hultgren SJ. Urinary tract infections: epidemiology, mechanisms of infection and treatment options. Nat Rev Microbiol. 2015;13(5):269-84.

2. Tandogdu Z, Wagenlehner FME. Global epidemiology of urinary tract infections. Curr Opin Infect Dis. 2016;29(1):73-9.

3. European Centre for Disease Prevention and Control. Point prevalence survey of healthcare-associated infections and antimicrobial use in European acute care hospitals 2011-2012. Available at: https://ecdc.europa.
eu/sites/portal/files/media/en/publications/Publications/healthcareassociated-infections-antimicrobial-use-PPS.pdf. Accessed 8 May, 2018.

4. European Centre for Disease Prevention and Control (ECDC). Point prevalence survey of healthcare-associated infections and antimicrobial use in European long-term care facilities. April-May 2013. Stockholm; 2014. Available at: https://ecdc.europa.eu/sites/portal/files/media/en/publications/ Publications/healthcare-associated-infections-point-prevalence-survey-longterm-care-facilities-2013.pdf. Accessed 8 May, 2018.

5. Levy SB, Marshall B. Antibacterial resistance worldwide: causes, challenges and responses. Nat Med. 2004;10(12s):S122-9.

6. Centers for Disease Control and Prevention (CDC). Antibiotic resistance threats in the United States, 2013. Available at: http://www.cdc.gov/ drugresistance/threat-report-2013/index.html. Accessed 8 May, 2018.

7. Mody L, Krein SL, Saint S, Min LC, Montoya A, Lansing B, et al. A targeted infection prevention intervention in nursing home residents with indwelling devices: a randomized clinical trial. JAMA Intern Med. 2015;175(5):714-23.

8. Surveillance Report. Surveillance of antimicrobial resistance in Europe 2016. Available at: https://ecdc.europa.eu/sites/portal/files/documents/AMRsurveillance-Europe-2016.pdf. Accessed 8, May, 2018.

9. Shaw E, Addy I, Stoddart M, Vank C, Grier S, Wiegand I, et al. Retrospective observational study to assess the clinical management and outcomes of hospitalised patients with complicated urinary tract infection in countries with high prevalence of multidrug resistant gram-negative bacteria (RESCUING). BMJ Open. 2016;6(7):e011500.

10. von Elm E, Altman DG, Egger M, Pocock SJ, Gøtzsche PC, Vandenbroucke JP, et al. Strengthening the reporting of observational studies in epidemiology (STROBE) statement: guidelines for reporting observational studies. BMJ. 2007;335(7624):806-8.

11. International Classification of Diseases, Ninth Revision, Clinical Modification, ICD-9-CM. Available at: https://www.cdc.gov/nchs/icd/icd9cm.htm. Accessed 14 Mar 2018.

12. International Classification of Diseases, Tenth Revision, Clinical Modification, ICD-10-CM. Available at: https://www.cdc.gov/nchs/icd/icd10cm.htm. Accessed 14 Mar 2018.

13. Complicated Urinary Tract Infections: Developing Drugs for Treatment. Guidance for Industry. U.S. Department of Health and Human Services, Food and Drug Administration Center for Drug Evaluation and Research (CDER), 2015. http://www.fda.gov/downloads/Drugs/Guidances/ucm070981.pdf. Accessed 8 May 2018

14. Magiorakos AP, Srinivasan A, Carey RB, Carmeli Y, Falagas ME, Giske CG, et al. Multidrug-resistant, extensively drug-resistant and pandrug-resistant bacteria: an international expert proposal for interim standard definitions for acquired resistance. Clin Microbiol Infect. 2012;18(3):268-81.

15. Nicolle LE, AMMI Canada Guidelines Committee* ACG. Complicated urinary tract infection in adults. Can J Infect Dis Med Microbiol. 2005;16:349-60.

16. Bader MS, Loeb M, Brooks AA. An update on the management of urinary tract infections in the era of antimicrobial resistance. Postgrad Med. 2017;129(2):242-58.

17. Bader MS, Hawboldt J, Brooks A. Management of complicated urinary tract infections in the era of antimicrobial resistance. Postgrad Med. 2010;122(6):7-15.

18. Bouchillon SK, Badal RE, Hoban DJ, Hawser SP. Antimicrobial susceptibility of inpatient urinary tract isolates of gram-negative bacilli in the United States: results from the study for monitoring antimicrobial resistance trends (SMART) program: 2009-2011. Clin Ther. 2013;35(6):872-7.

19. Hsueh P, Hoban DJ, Carmeli Y, Chen S, Desikan S, Alejandria M, et al. Consensus review of the epidemiology and appropriate antimicrobial therapy of complicated urinary tract infections in Asia-Pacific region. J Inf Secur. 2011;63(2):114-23.

20. Grundmann H, Glasner C, Albiger B, Aanensen DM, Tomlinson CT, Andrasević AT, et al. Occurrence of carbapenemase-producing Klebsiella pneumoniae and Escherichia coli in the European survey of carbapenemase-producing Enterobacteriaceae (EUSCAPE): a prospective, multinational study. Lancet Infect Dis. 2017;17(2):153-63.

21. Bonomo RA, Burd EM, Conly J, Limbago BM, Poirel L, Segre JA, et al. Carbapenemase-Producing Organisms: A Global Scourge. Clin Infect Dis. 2018;66(8):1290-7

22. Vasoo S, Barreto JN, Tosh PK. Emerging Issues in Gram-Negative Bacterial Resistance: An Update for the Practicing Clinician. Mayo Clin Proc. 2015; 90(3):395-403.

23. Exner M, Bhattacharya S, Christiansen B, Gebel J, Goroncy-Bermes P, Hartemann $P$, et al. Antibiotic resistance: What is so special about multidrug-resistant Gram-negative bacteria? GMS Hyg Infect Control. 2017;12:Doc05. 
24. Palacios-Baena ZR, Gutiérrez-Gutiérrez B, Calbo E, Almirante B, Viale P, Oliver $A$, et al. Empiric therapy with Carbapenem-sparing regimens for bloodstream infections due to extended-Spectrum $\beta$-lactamase-producing Enterobacteriaceae: results from the INCREMENT cohort. Clin Infect Dis. 2017;65(10):1615-23.

25. Zilberberg MD, Nathanson BH, Sulham K, Fan W, Shorr AF. Carbapenem resistance, inappropriate empiric treatment and outcomes among patients hospitalized with Enterobacteriaceae urinary tract infection, pneumonia and sepsis. BMC Infect Dis. 2017;17(1):279

26. Ostrowsky B, Banerjee R, Bonomo RA, Cosgrove SE, Davidson L, Doron S, et al. Infectious Diseases Physicians: Leading the Way in Antimicrobial Stewardship. Clin Infect Dis. 2018;66(7):995-1003.

27. Eliakim-Raz N, Babitch T, Shaw E, Addy I, Wiegand I, Vank C, et al. Risk factors for treatment failure and mortality among hospitalised patients with complicated urinary tract infection: a multicentre retrospective cohort study, RESCUING Study Group. Clin Infect Dis. 2018; https://doi.org/10.1093/cid/ciy418.

28. Magiorakos AP, Burns K, Rodríguez-Baño J, Borg M, Daikos G, Dumpis U, et al. Infection prevention and control measures and tools for the prevention of entry of carbapenem-resistant Enterobacteriaceae into healthcare settings: guidance from the European Centre for Disease Prevention and Control. Antimicrob Resist Infect Control. 2017;6:113.

29. Vazquez-Guillamet MC, Vazquez R, Micek ST, Kollef-Marin H. Predicting resistance to piperacillin-Tazobactam, Cefepime and Meropenem in septic patients with bloodstream infection due to gram-negative Bacteria. Clin Infect Dis. 2017;65(10):1607-14.

30. Bischoff S, Walter T, Gerigk M, Ebert M, Vogelmann R. Empiric antibiotic therapy in urinary tract infection in patients with risk factors for antibiotic resistance in a German emergency department. BMC Infect Dis. 2018;18(1):56.

31. Karlowsky JA, Lagacé-Wiens PRS, Simner PJ, DeCorby MR, Adam HJ, Walkty $A$, et al. Antimicrobial resistance in urinary tract pathogens in Canada from 2007 to 2009: CANWARD surveillance study. Antimicrob Agents Chemother. 2011;55(7):3169-75.

32. Hooton TM, Bradley SF, Cardenas DD, Colgan R, Geerlings SE, Rice JC, et al. Diagnosis, prevention, and treatment of catheter-associated urinary tract infection in adults: 2009 international clinical practice guidelines from the Infectious Diseases Society of America. Clin Infect Dis. 2010;50(5):625-63.

33. Pallett A, Hand K. Complicated urinary tract infections: Practical solutions for the treatment of multiresistant gram-negative bacteria. J Antimicrob Chemother. 2010;65 Suppl 3:iii25-33.

34. Tenke P, Kovacs B, Bjerklund Johansen TE, Matsumoto T, Tambyah PA, Naber KG. European and Asian guidelines on management and prevention of catheterassociated urinary tract infections. Int J Antimicrob Agents. 2008;31:68-78.

Ready to submit your research? Choose BMC and benefit from:

- fast, convenient online submission

- thorough peer review by experienced researchers in your field

- rapid publication on acceptance

- support for research data, including large and complex data types

- gold Open Access which fosters wider collaboration and increased citations

- maximum visibility for your research: over $100 \mathrm{M}$ website views per year

At BMC, research is always in progress.

Learn more biomedcentral.com/submissions 